\title{
Drug Encapsulated Nanomaterials as Carriers Used in Cardiology Field
}

\author{
ALINA COSTINA LUCA ${ }^{1,4}$, LUCIAN EVA ${ }^{2,5 *}$, LETITIA DOINA DUCEAC ${ }^{2,5 *}$, GETA MITREA ${ }^{3,6 *}$, \\ CONSTANTIN MARCU ${ }^{3,7}$, LIVIU STAFIE ${ }^{2,8}$, MADALINA IRINA CIUHODARU ${ }^{1,9}$, \\ IRINA MIHAELA CIOMAGA ${ }^{1,4}$, ELENA ROXANA BOGDAN GOROFTEI ${ }^{3,10}$, ELENA HANGANU ${ }^{1,4}$, \\ ALIN CONSTANTIN IORDACHE ${ }^{1,5}$ \\ ${ }^{1}$ Grigore T. Popa University of Medicine and Pharmacy of Iasi, 16 Universitatii Str., 700115, Iasi, Romania, Discipline of \\ Pediatric Surgery \\ ${ }^{2}$ Apollonia University of Iasi, Faculty of Dental Medicine, 11 Pacurari Str., 700511, Iasi, Romania \\ ${ }^{3}$ University Dunarea de Jos Faculty of Medicine and Pharmacy, 47 Domneasca Str., 800008, Galati, Romania \\ ${ }^{4}$ Sf. Maria Emergency Clinical Hospital for Children of Iasi, 62 Vasile Lupu Str., 700309, Iasi, Romania \\ ${ }^{5}$ Nicolae Oblu Neurosurgery Hospital of Iasi, 2 Ateneului 700309, Iasi,Romania \\ ${ }^{6}$ Sf. Ap. Andrei Emergency Clinical Hospital, 177 Brailei Str., 800578, Galati. Romania \\ ${ }^{7}$ Saarbrucken-Caritasklink St.Theresia University Hospital, Germany \\ ${ }^{8}$ Public Health Directorate of Iasi, 2-4 Vasile Conta, 7001016, Iasi, Romania \\ ${ }^{9}$ Elena Doamna Obstetrics and Gynecology Hospital of Iasi, 29 Elena Doamna Str., 700398, Iasi, Romania \\ ${ }^{10}$ Sf. Ioan, Emergency Clinical Hospital, 2 Gheorghe Asachi Str., 800494, Galati,m Romania
}

Pulmonary arterial hypertension is a disorder of high mortality being described by a progressive increase in pulmonary vascular strength leading to right ventricular damage and death. Endotelin-1 (ET-1) has an important pathogenic fuction in pulmonary hypertension. Bosentan is an oral ET-1 receptor antagonist that proved to be efficient at exercise tolerance in patients with pulmonary hypertension in functional class III and IV. The early trial registered for functional class II patients and evaluated hemodynamic at 6 month. Considerable side effects of bosentan comprise anemia, edema, and transaminase raise. Further studies were developed to determine optimum treatment strategy by eliminating adverse effects of bosentan. The major aim of this work was to prepare bosentan loaded nanoparticles by solvent evaporation technique for a sustained release of the drug by using ethyl cellulose as polymer. Drug and polymer were dissolved in ethanol at different drugpolymer ratios i.e. 1:2 and 1:3. Among the two formulations, 1:3 formulations were appreciated as the best formulation with better drug content (97.5\%) and incorporation efficiency (95.5\%).

Keywords: nanomaterials, pulmonary arterial hypertension, cardiological disorders, neurosurgical disorders, delivery systems, drug release

Pulmonary arterial hypertension is defined as changes that directly affect the pulmonary vessels including vasoconstriction, intravascular thrombosis, smooth muscle cell and endothelial proliferation $[1,2]$. It is an aggressive disease having a mean life of approximately three years after diagnosis. Symptoms consist of irregular breathing, fatigue, syncope, chest discomfort and peripheral edema that is predominant for females than for males [3-6]. Patients accusing pulmonary arterial hypertension are subjected to increased levels of Et-1which is a strong blood vessel constrictor met in lung tissues and plasma. Endothelin-1 decreases diameter of pulmonary arterial lumen, enhances pulmonary vascular toughness, reduces vascular bed reactivity finally elevating pulmonary arterial pressure $[7,8]$. Usually, for treatment of pulmonary arterial hypertension are used endothelin receptor antagonists.

Bosentan (figure 1) was the first one orally active for clinical investigation. It is a nonpeptide compound orally administrated defined by chemical formula $\mathrm{C}_{27} \mathrm{H}_{29} \mathrm{~N}_{5} \mathrm{O}_{6} \mathrm{~S}-\mathrm{H}_{2} \mathrm{O}$. Bosentan is administrated for pulmonary arterial hypertension treatment by blocking endothelin-1 molecule action being able to impede or change histological alteration produced by increased levels of Et-1.

This drug reaches peak plasma concentration 3 to 5 hours after consumption with an absolute bioavailability of $50 \%$ which is not affected by food. Bosentan is approximately $98 \%$ bound to albumin with a volume of distribution of $30 \mathrm{~L}$ and a terminal half-life of 5.4 hours. Steady-state plasma concentrations are reached in 5 days with a multiple dose regimen. The pharmacokinetics of bosentan are dose proportional up to $500 \mathrm{mg} /$ day; adult patients with PAH have a 2-fold 
increased exposure compared with healthy subjects and pediatric patients. Severe renal impairment (creatinine clearance 15 to $30 \mathrm{~mL} / \mathrm{min}$ ) and mild hepatic impairment do not have clinically relevant effects, but moderate to severe hepatic impairment are relative contraindications.

Metabolism of bosentan in the liver happens by the cytochrome p450 enzymes CYP2C9 and CYP3A4, with excretion of metabolites in the bile. One of the three metabolites identified, Ro 48-5033, could be pharmacologically active. Bosentan is an inducer of the CYP2C9 and CYP3A4 enzymes this explaining the increased clearance and reduced plasma levels of bosentan seen at steady-state. This induction alters the plasma levels of other compounds metabolized by these enzymes: glibenclamide, ciclosporin, simvastatin and warfarin. Specially, simvastatin levels may be reduced by 50\%; warfarin concentrations are lowed but no relevant changes in INR have been experienced [1-6].

Bosentan was studied in several clinical PAH trials with generally positive results. The first human study of bosentan treatment used an intravenous preparation which successfully decreased mPAP and PVR but it also provoked a drop in systemic arterial pressure and vascular resistance and afterwards an oral preparation has been favoured. After the benefit of oral bosentan in PAH was accredited in a randomized trial, subsequent studies have attempted to answer several outstanding questions which include: efficacy in subgroups of PAH and chronic thromboembolic disease, longterm effect on survival, safety and benefit when administrated in combination with other PAH therapies, and the value in milder illness [4-8].

In addition to the risk of inducing birth defects and of provoking liver damage, bosentan has a high risk of genetate pulmonary veno-occlusive disease, edema, decreasing sperm counts, and decreases in hemoglobin and hematocrit. Very common side effects, occurring in more than $10 \%$ of people, include elevated transaminases, headache and edema. Ordinary side effects, between $1 \%$ and $10 \%$ of people, include nasal congestion, hypersensitivity reactions, anemia, reduced hemoglobin, itchiness,skin inflammation, red skin, rashes, flushing, fainting, low blood pressure, heart palpitations, gastro-esophageal reflux disease, and diarrhea. When using a drug in situations where there may be less positive clinical effect, safety must come under closer close examination to guarantee that the risk-benefit ratio is still favourable. Bosentan offer benefit to those patients with PAH in FC II, but the relatively high incidence of adverse events may be prohibitive for some patients and clinicians. Moreover, bosentan, administered in cardiological pathology in both children and adults, improves the activity of the heart having beneficial implications for other specialties such as pregnant or gynecopathic with cardiological disorders - pulmonary hypertension, some patients who have to be operated for neurosurgical disorders and present as comorbid and have cardiological disorders [9-13].

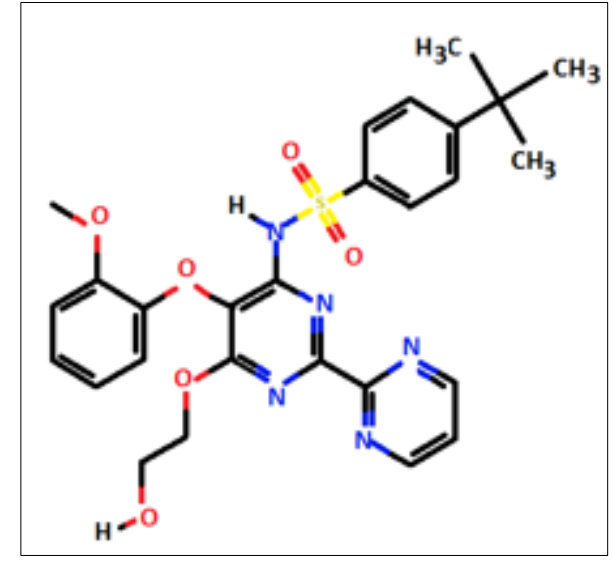

Fig. 1. Representation of bosentan chemical structure

Polymer nanoparticles emphasize an innovative non-invasive access for drug delivery uses. Nanoparticles major aim represents the transmission of the curative active molecule straightly to the target cell, tissue or organ. Numerous polymer compounds are useful for nanoparticles preparation; they consist of monomers that normally exist in the human body and hence simply evacuated without having toxic effects. Many drugs could be incorporated into the nanoparticles structure or physically distributed in their architecture [1-4].

For many years, pharmaceutical studies were concerned on the evolution of nanotechnology devices relevant in various domains of medicine, particularly in the area of drug carriage. The employment of biopolymer nanoparticles is an option that has a vast capability for targeted dispensation of drugs or other active molecules in the human body. Biopolymer nanoparticles may be successfully applied to assure bioactive molecules for in vivo and in vitro practicability. Moreover, nano-scaled biopolymers find uses in the area of cardiology and neurology. The chance of employ nanoparticles formed by eco-friendly and biocompatible polymers to carry drugs in damaged tissues constitutes a huge advantage by defeating many medical pains [3-10]. 
Biopolymer nanoparticles were used as carriers for drugs as they possess some advantages such as the administration of insoluble water drugs, the safety of unreliable compounds subjected to degradation. Nanoparticles may be charged with drugs either by encapsulation, adsorption or distribution into the polymer matrix. In the last few years, nanoparticles were used as delivery systems for drug transport to the affected organs. Nanoparticles are assumed by the target organ due to their very small size, good permeability and retention effect [6-11].

Using biodegradable nanoparticles as molecule vehicles is a big promising strategy for drug prolonged release systems. The basic request for a nano-biomaterial to be used in this direction is its biocompatibility, which means the facility to be metabolized with no injurious effect. These type of systems must be able to ensure properties such as the competence to pass thru anatomical barriers of human body, typically the blood-brain border or the ophthalmic border, holding the concentration of the drug over time and release the active molecule at the affected site [8-13].

Drug release from nanosized materials is controlled and sustained according to the nanoparticles composition and structure. Therefore, drug-loaded nanoparticles for local transport could optimize the efficacy and reduce the side effects of drugs [14-16]. Novel bosentan-loaded nanoparticles type formulation was obtained by solvent evaporation technique using ethyl cellulose as polymer and ethanol as solvent [17-22].

\section{Experimental part}

\section{Materials and methods}

Solvent evaporation technique was used to prepare bosentan loaded nanoparticles which involves a few steps. First one refers to emulsification of the ethyl cellulose solution into an aqueous phase. The second step implies the evaporation of polymer solvent causing polymer precipitation as nanospheres. The obtained nanoparticles were separated by centrifugation, washed vigorously with deionised water and lyophilized for storage. Furthermore, active substance bosentan and ethyl cellulose were dissolved in ethanol at varied ratios of drug-polymer,1:2 and 1:3 respectively, then sonicated for 10 minutes. The obtained organic dispersion was emulsified by stirring at $700 \mathrm{rpm}$ into an external phase containing tween-20 reagent at room temperature. The obtained organic phase-aqueous phase ratio of 1:10 was maintained by addition of organic phase drop wise to the aqueous phase at a constant rate. Ethanol was evaporated by stirring of the oil in water emulsion. Finally, the resultant dispersion was collected using a vacuum evaporator and preserved for drying.

\section{Results and discussions}

The obtained samples of bosentan loaded ethyl cellulose nanoparticles were assessed for novel formulation morphology, drug content, incorporation efficiency, loading capacity and drug release.

Scanning electron microscopy was used to analyze the nano-sized surface and morphological features of the pure drug and the drug loaded nanoparticles. Bosentan (figure 2A) was distinguished by the presence of oral, nano-sized, unequal, rough and fragmented particles.
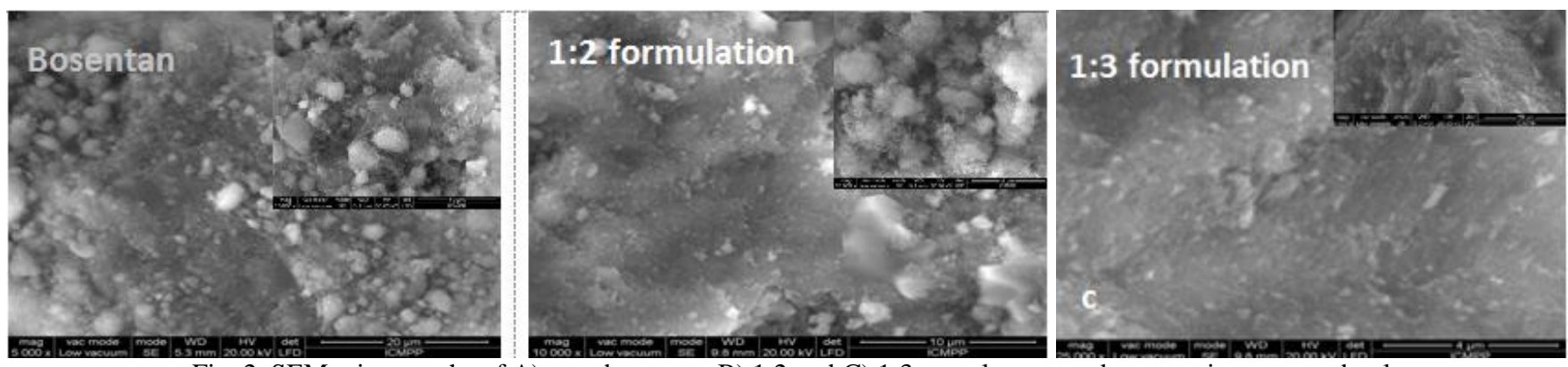

Fig. 2. SEM micrographs of A) pure bosentan, B) 1:2 and C) 1:3 complexes type bosentan incorporated polymer nanoparticles at different magnitudes

Bosentan loaded ethyl cellulose nanoparticles micrographs exhibited the characteristic morphology as irregular fragments of amorphous ensembles and the initial morphologies of pristine bosentan attenuated (figure $2 \mathrm{~B}$ and $2 \mathrm{C}$ ). Bosentan and drug loaded polymer micrographs suggested the formation of novel formulation type drug loaded nanoparticles in the solid state.

Drug content evaluation (figure 3) was accomplished by transferring exactly $50 \mathrm{mg}$ of the bosentan in $50 \mathrm{ml}$ methanol under magnetic stirring at $700 \mathrm{rpm}$ for 5 hours. The obtained solution was filtrated then the bosentan content was determined using UV-VIS spectrophotometry at a wavelength of $272 \mathrm{~nm}$. 
Entrapment efficiency (figure 4) was determined by the amount of bosentan contained in the novel formulation. The drug comprised with naoparticles can be either in the form of incorporation in the ethyl cellulose matrix or/and adsorption onto the polymer surface.

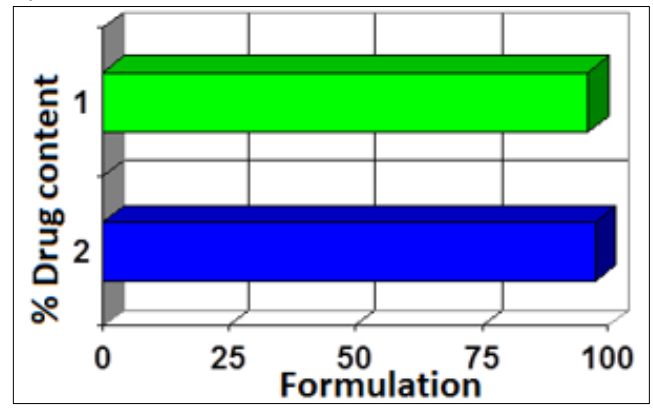
Fig. 3. Comparison of drug content of the two formulations $1: 2$ and 1:3.

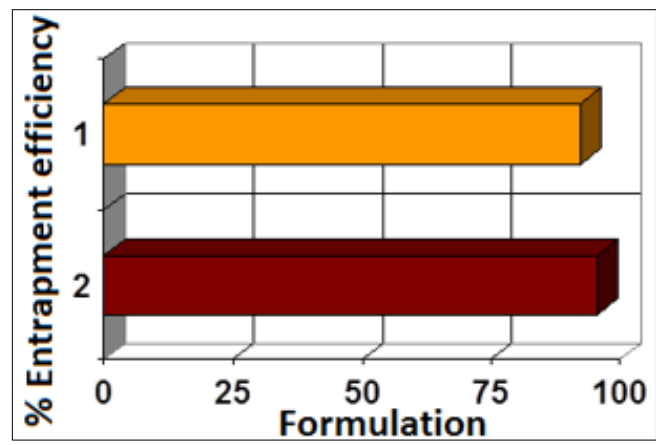

Fig. 4. Comparison of incorporation efficiency of the two formulations $1: 2$ and $1: 3$.

Incorporation efficiency was obtained by centrifuging the samples containing $50 \mathrm{mg}$ of the drug in $50 \mathrm{ml}$ phosphate buffer solution of $\mathrm{Ph} 6.8$ at $15000 \mathrm{rpm}$ at $5^{\circ} \mathrm{C}$ for 50 minutes.

Loading capacity (figure 5) denotes the performance of the ethyl cellulose to load bosentan. Results showed that among the two formulations, 1:3 formulation revealed highest loading capacity.

In vitro drug release profile (figure 7) obtained using orbitary shaker for 6 hours pointed out that the maximum bosentan release was of 1:3 formulation $(25 \%)$.

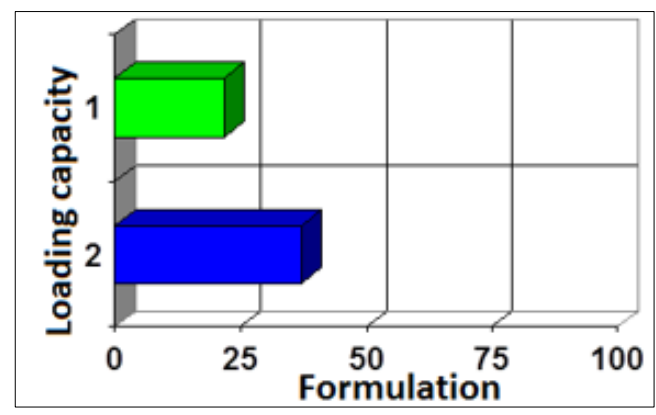

Fig. 5. Comparison of loading capacity of the two formulations $1: 2$ and $1: 3$.

For samples yield evaluation, the obtained drug loaded nanoparticles were dried and the percentage yield was calculated for both formulations. The highest percentage (90\%) was for the 1:3 formultion as shown in figure 6.

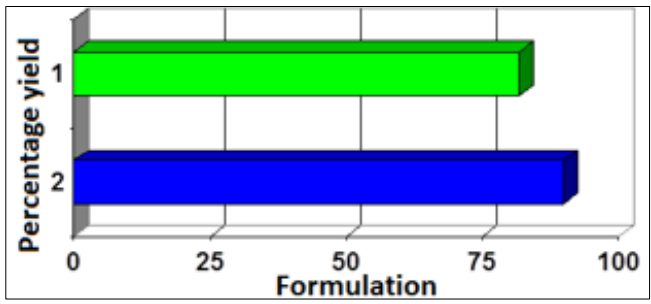

Fig. 6. Comparison of $\%$ yield of the two formulations $1: 2$ and 1:3

Drug release kinetics was established by performing in vitro dissolution investigations using an orbitary shaker. Precisely weighed $50 \mathrm{mg}$ of each formulation was added to $50 \mathrm{~mL}$ phosphate buffer solution at $\mathrm{Ph} 6.8$ and putted in the 
orbitary shaker at $100 \mathrm{rpm}$ at a constant temperature of $37^{\circ} \mathrm{C} .2 \mathrm{~mL}$ of samples were removed at clearly defined time intervals and substituted with buffer solution of the same volume. The amount of bosentan release was obtained from the absorbance values at $272 \mathrm{~nm}$ wavelength using UV-VIS spectrophotometer.

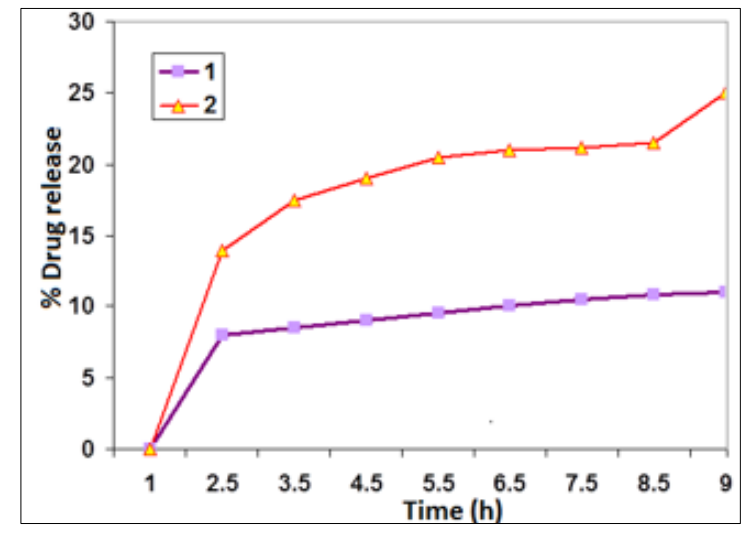

Fig. 7. Comparison of $\%$ cumulative drug release of the two formulations $1: 2$ and $1: 3$

In this work, drug loaded polymer nanoparticles were prepared by varying bosentan to ethyl cellulose ratio i.e. 1:2 and 1:3. The best formulation with better features was found 1:3 compared to 1:2 showing high drug content, loading capacity, and incorporation of the drug, high percentage yield and better controlled drug release.

\section{Conclusions}

Bosentan is an effective drug at increasing exercise tolerance for pulmonary arterial hypertension patients belonging to functional class III and IV. Given the probability that the treatment for functional class II patients can be necessary for several years, the ideal drug for pulmonary hypertension would be one with no serious adverse effects. Polymer nanoparticles type ethyl cellulose was used as drug carrier. There is why bosentan loaded nanoparticles could reduce side effects due to the sustained release from drug carriers. In this work bosentan loaded nano-sized biocompatible materials were successfully prepared in order to constitute an advantage in the management of pulmonary arterial hypertension. This fact offers new perspectives for pharmacokinetic and tolerance researches regarding bosentan loaded delivery nanosystems in human. Bosentan incorporated nanoparticles might improve the efficacy and minimize the adverse effects of the drug.

\section{References}

1.SIMONNEAU, G., GALIÈ, N., RUBIN, L.J., LANGLEBEN, D., SEEGER, W., DOMENIGHETTI, G., GIBBS, S., LEBREC, D., SPEICH, R., BEGHETTI, M., RICH, S., FISHMAN, A., J Am Coll Cardiol., 43(S), 2004, p. 5S.

2.FARBER, H.W., LOSCALZO, J., New Engl J. Med., 351, 2004, p. 1655.

3.RUBIN, L.J., N Engl J Med, 336, 1997, p. 111.

4.DINGEMANSE, J., VAN, GIERSBERGEN, P.L., Clin Pharm, 43, 2004, p. 1089.

5.BENZA, R.L., MILLER, D.P., BARST, R.J., BADESCH, D.B., FROST, A.E., MCGOON, M.D., Registry. Chest, 142,2012 , p. 448.

6.SAIGAL, A., NG, W.K., TAN, R.B., Int J Pharm, 450, 2013, p. 114.

7.WILLIAMSON, D.J., WALLMAN, L.L., JONES, R., KEOGH, A.M., SCROOPE, F., PENNY, R., WEBER, C., MACDONALD, P.S., Circulation, 102, 2000, p. 411.

8.NAHAR, K., ABSAR, S., PATEL, B., AHSAN, F., Int J Pharm, 464, 2014, p. 185.

9.CLOZEL, M., BREU, V., GRAY, G.A., KALINA, B., LÖFFLER, B.M., BURRI, K., CASSAL, J.M., HIRTH, G., MÜLLER, M., NEIDHART, W., J Pharmacol Exp Ther , 270, 1994, p. 228.

10.ROUX, S., BREU, V., ERTEL, S.I., CLOZEL, M., J Mol Med, 77, 1999, p. 364.

11.OHLSTEIN, E.H., DOUGLAS, S.A., Drug Dev Res, 29, 1993, p. 108.

12.VACHIERY, J.L., SIMONNEAU, G., Eur Respir Rev, 19, p. 2010, p. 279.

13.GRANTON, J., MERCIER, O., DE, PERROT, M., Semin Respir Crit Care Med, 34, 2013, p. 700.

14.KIM, B.Y., RUTKA, J.T., CHAN,W.C., Engl. J. Med., 363, 2010, p. 2434.

15.MATOBA, T., EGASHIRA, K., Int. Heart J., 55, 2014, p. 281.

16.VAIDYA, B., GUPTA, V., J. Control. Release, 211, 2015, p. 118.

17.SENTHILKUMAR, B., RAJASEKARAN, R., Journal of Bionanosci, 10(1), 2016, p.1.

18.REIS, C.P., NEUFELD, R.J., RIBEIRO, A.J., VEIGA, F., Nanomedicine, 2(1), 2006, p. 8.

19.QUINTANAR-GUERRERO, D., FESSI, H., ALLE'MANN, E., DOELKER, E., Int J Pharm, 143(2), 1996, p. 133.

20.QUINTANAR-GUERRERO, D., ALLÉMANN, E., FESSI, H., DOELKER, E., Drug Dev Ind Pharm 24, 1998 , p. 1113.

21.LIMAYEM, A., MOHAPATRA, S.S., HALLER, E., Nanomed Nanotechnol, 1(2), 2016, p. 000107.

22.QUIÑONES, J.P., KJEMS, J., YANG, C., PENICHE, C., BRÜGGEMANN, O., Nanomed Nanotechnol, 2(3), 2017, p. 000128. 
Manuscript received: 10.02 .2020 